\title{
ATP-binding cassette transporter enhances tolerance to DDT in Tetrahymena
}

\author{
NING YingZhi ${ }^{1}$, DANG Huai ${ }^{1}$, LIU GuangLong ${ }^{1}$, XIONG Jie $^{2}$, YUAN DongXia ${ }^{2}$, \\ FENG LiFang $^{3 *} \&$ MIAO Wei ${ }^{2 *}$ \\ ${ }^{1}$ College of Life Sciences, Northwest Normal University, Lanzhou 730070, China; \\ ${ }^{2}$ Key Laboratory of Aquatic Biodiversity and Conservation, Institute of Hydrobiology, Chinese Academy of Sciences, \\ Wuhan 430072, China: \\ ${ }^{3}$ Key Laboratory of Food Safety in Zhejiang Province, College of Food Science and Biotechnology, Zhejiang Gongshang University, \\ Hangzhou 310018, China
}

Received June 17, 2014; accepted August 4, 2014; published online September 23, 2014

\begin{abstract}
The reuse of dichlorodiphenyltrichloroethane (DDT) as an indoor residual spray was permitted by the World Health Organization in 2007, and approximately 14 countries still use DDT to control disease vectors. The extensive exposure of insects to DDT has resulted in the emergence of DDT resistance, especially in mosquitoes, and the mechanism for this resistance in mosquitoes has been widely reported. Spraying can also introduce DDT directly into surface water, and DDT can subsequently accumulate in microorganisms, but the mechanism for the resistance to DDT degradation in microorganisms is unclear. Using whole-genome microarray analysis, we detected an $a b c b 15$ gene that was up-regulated in a specific manner by DDT treatment in T. thermophile. The deduced ABCB15 peptide sequence had two transmembrane domains (TMDs) and two nucleotide-binding domains (NBDs) to form the structure TMD-NBD-TMD-NBD, and each NBD contained three conserved motifs: Walker-A, C-loop, and Walker-B, which indicated the T. thermophila abcb15 was a typical ABC transporter gene. The expression of $\mathrm{ABCB} 15$ fused with a C-terminal green fluorescent protein was found to be on the periphery of the cell, suggesting that $\mathrm{ABCB} 15$ was a membrane pump protein. In addition, cells with abcb15 partially knocked down (abcb15-KD) grew slower than wild-type cells in the presence of $256 \mathrm{mg} \mathrm{L}^{-1}$ DDT, indicating the tolerance of abcb15-KD strain to DDT exposure was decreased. Thus, we suggest that in Tetrahymena, the membrane pump protein encoded by ABCT gene abcb15 can enhance the tolerance to DDT and protect cells from this exogenous toxin by efficiently pumping it to the extracellular space.
\end{abstract}

Tetrahymena, DDT, ATP-binding cassette transporter, tolerance

Citation: $\quad$ Ning YZ, Dang H, Liu GL, Xiong J, Yuan DX, Feng LF, Miao W. ATP-binding cassette transporter enhances tolerance to DDT in Tetrahymena. Sci China Life Sci, 2015, 58: 297-304, doi: 10.1007/s11427-014-4743-x

Dichlorodiphenyltrichloroethane (DDT), an organochlorine pesticide, was used to control insects that carried diseases (e.g., malaria and typhus) and destroyed agricultural crops worldwide from the 1940s to the 1960s. With high lipophilicity and a long half-life, DDT sprayed into the environment and accumulated in organisms, creates significant threats to wildlife and human health. For example, DDT has

*Corresponding author (email: miaowei@ihb.ac.cn; fenglifang@ mail.zjgsu.edu.cn) been implicated in a wide range of adverse effects on humans, such as liver cancer, pancreatic cancer, breast cancer, diabetes, pregnancy loss, poor sperm quality, and neurodevelopmental toxicity in children [1]. Thus, DDT was banned in many countries in the 1970 s and was one of the 12 recognized persistent organic pollutants at the Stockholm Convention in 2001. However, currently DDT remains to be a significant threat to the health of wildlife and humans for two reasons: first, the prevalence of malaria markedly in- 
creased in some African countries after the banning of DDT. Therefore, the World Health Organization suggested the reuse of this pesticide in some areas in September 2006 [2]. Second, DDT concentrations are still growing in the northern hemisphere through long-range oceanic and atmospheric transport [3].

The extensive exposure of insects to DDT has resulted in the emergence of DDT resistance, especially in the mosquito. Currently, it is thought that the DDT resistance in the mosquito is mediated through two main mechanisms: first, the DDT resistance could be caused by the mutations in DDT target sites, such as the voltage-gated sodium channel, insensitive acetylcholinesterases, and the neurotransmitter $\gamma$-aminobutyric acid, which lead to low DDT binding capacity [4]. Second, the DDT resistance in mosquito could be due to alterations in the activities or levels of detoxification proteins, such as carboxylesterases, cytochrome P450 monooxygenases, and glutathione $S$-transferases. Since sprayed DDT can be released onto surface water directly or indirectly through dry and wet deposition from the atmosphere [5], microorganisms can also adapt to the presence of DDT under its long-term exposure, thus allowing its accumulation and serial transfer to higher levels in the food chain [6]. Therefore, analysis of the metabolism of DDT in aquatic microorganism (e.g., protozoa and bacteria) is very important.

Tetrahymena is a free-living ciliated protozoan distributed in freshwater environments around the world, and it has the conserved genes and basic metabolic pathways of a metazoan [7]. Studies in Tetrahymena have led to numerous scientific breakthroughs, and a number of molecular genetic technologies and genomic resources have been developed [8-10]. Using a microarray technique, we recently identified genes that were differentially expressed in Tetrahymena thermophila after exposure to DDT, and we found that an ATP-binding cassette transporter (ABCT) gene ( $a b c b 15)$ belonging to the B family may be associated with the robust DDT resistance of $T$. thermophile [11]. In the present study, the function of $a b c b 15$ was analyzed to elucidate the mechanism of tolerance to DDT in T. thermophile.

\section{Materials and methods}

\subsection{Cell culture and toxicity experiments}

Two inbred strains of $T$. thermophila (SB210 and CU428) were kindly provided by Dr. Eduardo Orias (University of California, USA). Cells were grown as axenic cultures in super proteose peptone medium. The stock solutions of DDT and 2,3,7,8-Tetrachlorodibenzo-p-dioxin (TCDD) were described in our previous studies $[11,12]$. For toxicity analyses of growing cells, cells in the mid-logarithmic growth phase $\left(\sim 3 \times 10^{5}\right.$ cells $\left.\mathrm{mL}^{-1}\right)$ were inoculated into the treatment medium of $4 \mathrm{mg} \mathrm{L}^{-1}$ DDT and $1 \mu \mathrm{g} \mathrm{L}^{-1}$ TCDD for $24 \mathrm{~h}$.

1.2 RNA extraction, cDNA synthesis, mRNA purification, SMART cDNA library construction, and $5^{\prime}$ and $3^{\prime}$ RACE cloning

Cells were collected after exposure to the medium for toxicity studies, and their total RNA was extracted using an RNeasy Protect Cell Mini Kit (Qiagen, Germany). RNA quality was monitored using spectrophotometry (Malcom, Japan) and electrophoresis. Total RNA was digested with DNase (Promega, USA) and reverse transcribed into double-stranded cDNA using M-MLV reverse transcriptase RNase $\mathrm{H}^{+}$(TOYOBO, Japan) [13].

The cultures $(5 \mathrm{~mL})$ were incubated in the presence of 4 $\mathrm{mg} \mathrm{L}^{-1}$ DDT for $24 \mathrm{~h}$. Total RNA was then extracted and purified using PolyAtract mRNA isolation systems (Promega), and the purified mRNA was used to construct a SMART cDNA library (Clontech, USA) [14].

Using the SMART cDNA library as a template, $5^{\prime}$ - and 3'-untranslated regions (UTRs) (5' and 3' rapid amplification of cDNA ends, RACE) of the $a b c b 15$ gene were PCR amplified using the primers 5' UT_f (GCAGTGGTATCAACGCAGAGTG) and abcb15_r (GTACCCACTGAAAGGATAACAAACTC), and 3' UT_r (GGCGGCCGACATGTTTTTTT) and abcb15_f (AGAATACAAATGTGAAGTAAGCCTAAG), respectively [15]. The reactions were performed in a $25-\mu \mathrm{L}$ mixture, containing $2.5 \mu \mathrm{L}$ of $10 \times$ buffer, $0.5 \mu \mathrm{L}$ of $10 \mathrm{mmol} \mathrm{L}^{-1} \mathrm{dNTP}$ (Fermentas, Lithuania), $0.4 \mu \mathrm{mol} \mathrm{L} \mathrm{L}^{-1}$ primers, 1 unit Taq Polymerase (BD advantage, USA), and $0.5 \mu \mathrm{L}$ of template. The PCR cycling conditions were as follows: denaturation at $94^{\circ} \mathrm{C}$ for $5 \mathrm{~min}$; amplification for 35 cycles of $30 \mathrm{~s}$ at $94^{\circ} \mathrm{C}, 30 \mathrm{~s}$ at $62^{\circ} \mathrm{C}$, and $60 \mathrm{~s}$ at $72^{\circ} \mathrm{C}$; extension at $72^{\circ} \mathrm{C}$ for $10 \mathrm{~min}$. The PCR products were analyzed using electrophoresis with agarose gels and purified with a GlassMilk kit (Biostar, China). The purified products were ligated into pGEMT-Vectors (Promega) and transformed into Escherichia coli DH-5a cells. Positively screened clones were sequenced with an ABI model 377 Stretch automated DNA sequencer (PE Applied Biosystems, USA).

\subsection{Subcellular localization of ABCB15}

\subsubsection{Predictions using biosoftware}

The subcellular localization of ABCB15 was predicted from its derived protein using WoLF PSORT (http://wolfpsort. org/) [16] and TargetP 1.1 (http://www.cbs.dtu.dk/services /TargetP/) [17] with default parameters.

\subsubsection{In situ localization}

For in situ localization, ABCB15 was tagged with green 
fluorescent protein (GFP) in vivo. Using cDNA (prepared in 1.2 ) as a template, the $a b c b 15$ gene was amplified with PCR using primers TOPO_ABCB15_f (CACCTAAAAAATGGAAGAAAAGTCATTATA) and TOPO_ABCB15_r (GCAATTCTAATTTTAACTTTCCTGC), and the product was cloned into $\mathrm{pENTR}^{\mathrm{TM}} / \mathrm{D}-\mathrm{TOPO}$ (Invitrogen, USA) to form the plasmid pENTR_ABCB15. After the LR recombination reaction, pENTR_CYP5013C2 was introduced into pICC_ gtw vectors (kindly provided by Professor Douglas L. Chalker, University of Washington, USA) to form the recombinant plasmid PICC_ABCB15 by replacing the $c c d B$ coding region [18] (Figure 1). The reaction was performed in a $5-\mu \mathrm{L}$ mix, containing $3 \mu \mathrm{L}$ of pENTR_ABCB15 plasmid, $1 \mu \mathrm{L}$ of PICC_gtw, and $1 \mu \mathrm{L}$ of Gateway LR Clonase II Enzyme Mix (Invitrogen).

The recombinant plasmid was introduced into Tetrahymena by conjugative electrotransformation [19]. Transformants were selected using serial transfers in $1 \times$ super proteose peptone medium supplemented with increasing amounts (from 100 to $1000 \mathrm{mg} \mathrm{L}^{-1}$ ) of paromomycin (Sigma, USA) and then verified with whole cell PCR using the primers TOPO_ABCB15_f (CACCTAAAAAATGGAAGAAAAGTCATTATA) and GFP_r (ATGTGGCTCCTTATCTTACCCTA) that flanked the $a b c b 15$ and $g f p$ genes. After adding $5 \mathrm{mg} \mathrm{L}^{-1} \mathrm{CdCl}_{2}$ to $2 \mathrm{~mL}$ of transformed cells $\left(3 \times 10^{5}\right.$ cells $\left.\mathrm{mL}^{-1}\right)$, the $m t t l$ promoter was induced. Three hours later, the cells were concentrated to a final concentration of $1 \mathrm{mgL}^{-1}$, fixed in $2 \%$ paraformaldehyde, and stained with 4',6-diamidino-2-phenylindole (DAPI, Sigma). An Axioplan 2 Imaging (Zeiss, Germany) microscope was used to detect the localization of the GFP fusion protein.

\subsection{Construction of the ABCB15 knockdown strain}

Using total genomic DNA as template, the 5' and $3^{\prime}$ flanking regions were amplified with $\mathrm{PCR}$ using primers abcb15_5f (TTATTAAAGTTATGATTGTCCTGCTA) and abcb15neo_5r (GTGTATTTAAATTAAAGGAGTTATTCTCTGAAACCCATTAGTCAAAGTAG, overlapping sequences are underlined), and abcb15neo_3f (CAAATTTTTACTGGAAAAATGCATGGAAAATGGCAACATAG TCG, overlapping sequences are underlined) and abcb15_ 3r (ACTTAAAACCAAGCTCAAAAGACAG), respectively. The neo4 cassette DNA was obtained with PCR amplification using the plasmid pNeo4 (kindly provided by Professor Mochizuki, Institute of Molecular Biotechnology of the Austrian Academy of Sciences, Austria) as a template and primers neo4_f (GAATAACTCCTTTAATTTAAATACAC) and neo4_r (GCATTTTTCCAGTAAAAATTTG) [20]. The 5' flanking region, neo4 cassette, and 3' flanking region were linked through overlapping PCR using the primers abcb15nest_f (AAAGTTATGATTGTCCTGCTATTA) and abcb15nest_r (TAAAACCAAGCTCAAAAGACAG) to form the knockdown target construct. The PCR cycling conditions were as follows: denaturation for $10 \mathrm{~min}$ at $94^{\circ} \mathrm{C}$; amplification for 35 cycles of $30 \mathrm{~s}$ at $94^{\circ} \mathrm{C}, 1 \mathrm{~min}$ at $50^{\circ} \mathrm{C}$, and $5 \mathrm{~min}$ at $72^{\circ} \mathrm{C}$; extension for $10 \mathrm{~min}$ at $72^{\circ} \mathrm{C}$.

The PCR product was analyzed using electrophoresis with agarose gels and purified with a GlassMilk kit (Biostar). The purified product was transformed into starved B2086 cells using a Bio-Rad biolistic gun [21]. Transformants were selected with electrotransformation. During the selection process, endogenous macronuclear $a b c b 15$

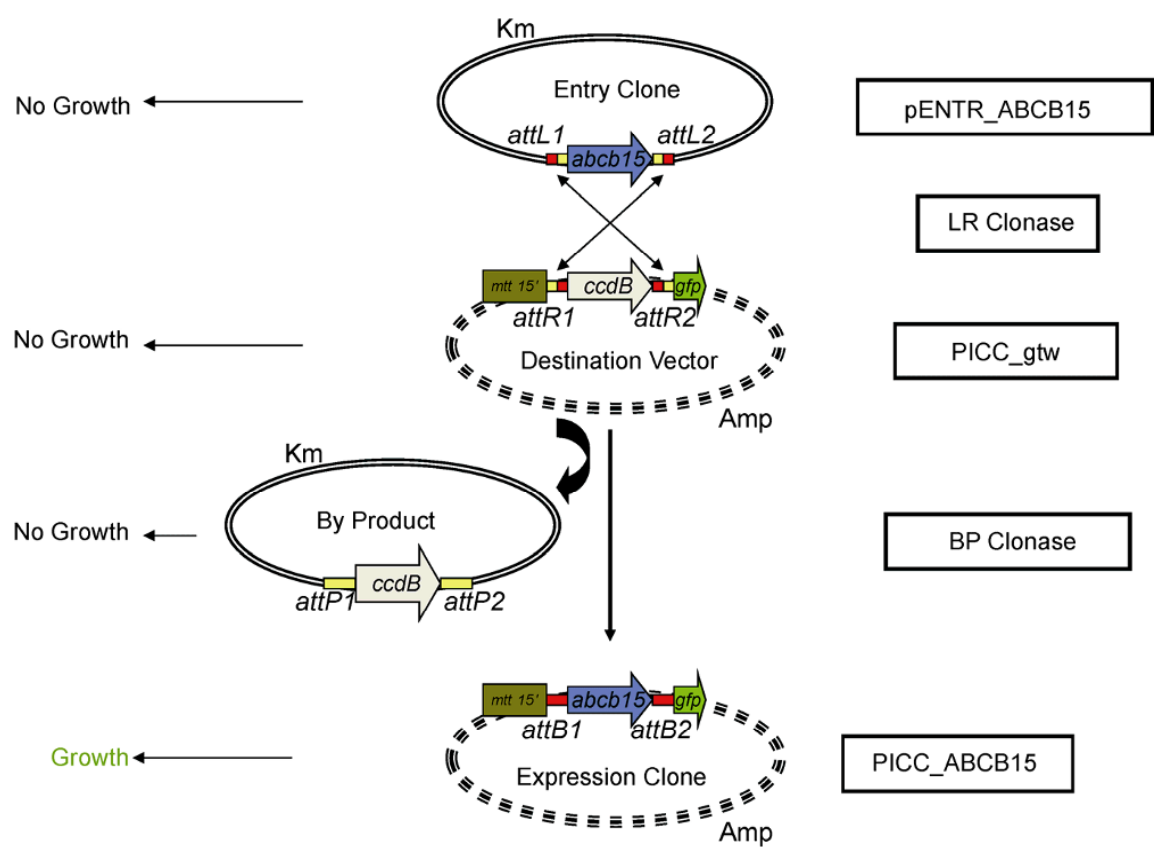

Figure 1 The target gene $a b c b 15$ is placed into the PICC_gtw vector using the Gateway homologous recombination system. 
alleles were partially replaced with the abcb15 knockdown alleles using phenotypic assortment (Figure 2). The knockdown strain of $a b c b 15$ was named $a b c b 15-\mathrm{KD}$ strain.

Cells with $a b c b 15-\mathrm{KD}$ and wild-type strains in mid-logarithmic growth phase $\left(\sim 3 \times 10^{5}\right.$ cells $\left.\mathrm{mL}^{-1}\right)$ were inoculated into the treatment medium of $4 \mathrm{mg} \mathrm{L}^{-1}$ DDT for $24 \mathrm{~h}$, and total RNA was then extracted and subsequently reverse-transcribed into cDNA. The presence of the $a b c b 15$ knockdown construct was detected with real-time PCR using the template of the above cDNA and the primers abcb15_f and abcb1_r; the 18s rRNA gene, as an endogenous control, was amplified with primers of $18 \mathrm{~s} \_\mathrm{f}$ (CCTGGGAAGGTACGGGTAAT) and 18s_r (AAGGTTCACCAGACCATTCG). Real-time PCR reactions and the calculations of the relative expression levels of $a b c b 15$ were performed as described in our previous study [13].

\subsection{Growth models in T. thermophila}

Cells in the mid-logarithmic growth phase $\left(\sim 3 \times 10^{5}\right.$ cells $\left.\mathrm{mL}^{-1}\right)$ of the B2086 or $a b c b 15-\mathrm{KD}$ strains were inoculated into $20 \mathrm{~mL}$ of fresh super proteose peptone medium, and the final density was $\sim 1 \times 10^{4}$ cells $\mathrm{mL}^{-1}$, with $256 \mathrm{mg} \mathrm{L}^{-1}$ DDT and $0.05 \mu \mathrm{g} \mathrm{mL}^{-1} \mathrm{CdCl}_{2}$. The samples were collected at 3 , $4.5,8,10.5$, and $14.5 \mathrm{~h}$, and the cells were counted (Beckman Coulter, USA). To adjust to the new environment after inoculation, cells underwent a lag phase of 0-4.5 h. During this transition, the first-order growth model was postulated as $N_{B}=N_{A} \times \exp (k \times t)$, where $N_{B}$ is cell density in stage $\mathrm{B}, N_{A}$ is cell density in stage $\mathrm{A}, k$ is the growth rate parameter $\left(\mathrm{h}^{-1}\right)$, and $t$ is a specific time. Once activated, cells increased by binary division, with the corresponding growth model as $N_{C}=N_{B} \times 2^{t / G}$, where $G$ is generation time (h) and $N_{C}$ is cell density in stage $\mathrm{C}$ [22].

\section{Results}

\subsection{The characteristics of $T$. thermophila abcb15 gene}

Our sequencing results showed that the full-length of abcb15 gene in T. thermophila was $4937 \mathrm{bp}$, containing 9 exons and 8 introns (Figure 3). The mRNA length was 4157 bp, with an open reading frame (ORF) of 3957 nucleotides for 1318 aa. There were 49 nucleotides in the $5^{\prime}$-UTR and 151 nucleotides in the $3^{\prime}$-UTR, including a conserved consensus polyadenylation signal element AATAAA [23]. The deduced ABCB15 peptide sequence had two transmembrane domains (TMDs) and two nucleotide-binding domains (NBDs) to form the structure TMD-NBD-TMD-NBD [24]. In addition, each NBD contained three conserved motifs: Walker-A, C-loop, and Walker-B (Figure 3). All these results indicate that $a b c b 15$ in $T$. thermophila is a typical $\mathrm{ABC}$ transporter gene.

\subsection{The ABC transporter gene abcb15 is up-regulated by DDT in $T$. thermophila}

It has been shown by a microarray study that 75 genes are up-regulated more than two fold by the DDT treatment, including $\mathrm{ABC}$ transporter genes related to DDT detoxification [11]. When we carefully examined three physiological/developmental stages (growth, starvation, and conjugation) and different (DDT or TCDD) treatments, we found that only DDT treatment resulted in a remarkably increase in the expression of $a b c b 15$ (Figure 4). Combined with the microarray data at different life cycle stages [8], we conclude that abcb15 (TTHERM_00240450) was up-regulated in a specific manner by DDT treatment in T. thermophile.

\subsection{ABCB15 protein is localized on the cell membrane in T. thermophila}

Based on the WoLF PSORT and TargetP 1.1 software computational analyses, ABCB15 was localized on the cell membrane. This localization is further confirmed by fluorescent studies. When $a b c b 15$ was fused with C-terminal GFP, the expression of this ABC transporter chimeric protein under $\mathrm{CdCl}_{2}$-inducing conditions was found to be on the periphery of the cell (Figure 5).

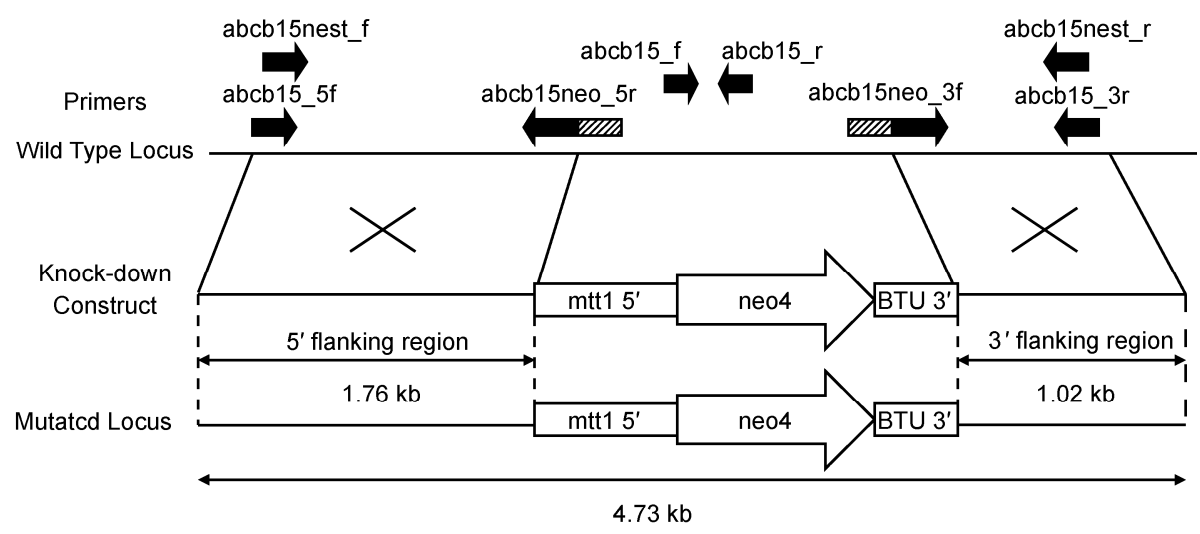

Figure 2 Schematic representation of the targeted $a b c b 15$ knockdown construct and the strategy for in vivo replacement. 
GAATAGCTTAAACATATATAATCGATTAAACTGAAAGCATATATAAAAA aTGG M $E \begin{array}{llllllllllllllllllll} & E & E & K & S & L & Q & N & L & E & K & P & T & Q & N & Y & E & D & Q & E\end{array}$ GTGGCAGATGGTAAATACCATTTTGGCATCTATTTMAACTAGCATCGAAAACTGATATC

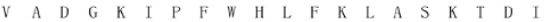
ATTTTAGTTGTTTTAGGAAGCTTAGCTTCTGTTATAAATGGTTGTCTGTAGCCACTTTTT I $L$\begin{tabular}{llllllllllllllllll}
\hline & $V$ & $L$ & $G$ & $S$ & $L$ & $A$ & $S$ & $V$ & $I$ & $N$ & $G$ & $C$ & $L$ & $Q$ & $P$ & $L$ & $F$ \\
\hline
\end{tabular} GGTCTACTATTTGGTGAAATGGCATAAAAATTTAGCCCTGGATATAGCGCCGATGCAGTT

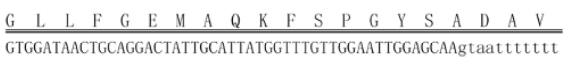
\begin{tabular}{lllllllllllllllll}
$V$ & $D$ & $N$ & $C$ & $R$ & $T$ & $I$ & $A$ & $L$ & $\mathbb{W}$ & $F$ & $V$ & $G$ & $I$ & $G$ & $A$ & I \\
\hline
\end{tabular}

tagttgataattttgatctcatcataatggtagagataagaggataaaatatgcctaca tcttt taataat $t$ tg t taagtatatatcaaact ttactatt $t$ tatattaaaagTATCA

TTTATACTTTCAATCTTTATGATGTACTTTTGGATCAGGGTGGGTTAGAGATAGGCAATA \begin{tabular}{llllllllllllllllllll}
$F$ & $I$ & $L$ & $S$ & $I$ & $F$ & $M$ & $M$ & $Y$ & $F$ & $\mathbb{W}$ & $I$ & $S$ & $V$ & $G$ & $Q$ & $R$ & $Q$ & $A$ & $I$ \\
\hline
\end{tabular} AutTTAGATTAGaGTACTTTAaGTCTTTGCTCAAATAAGAagTAGGTTATTTTGATTAA

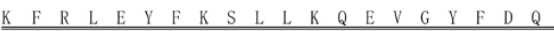
ATTTAGgCAAATGaGCTTTCATCTAAaGTATCTACTGAATGCTTCAAAATATAGTCAGCA TTGGGaGaAaAaCATGCATCTTTATTTATTCATTGAGTATGTTTATTGGTAGTCTTATA \begin{tabular}{llllllllllllllllllll} 
L & $G$ & E & K & T & C & I & F & I & Y & S & L & $S$ & M & F & I & G & S & L & I \\
\hline ATAGCCTTTATTAGGGGATGGCAAATTGCTCTTGTGGCAATTGCTGTACACCACTTATA
\end{tabular}

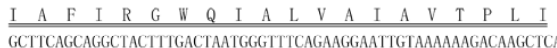

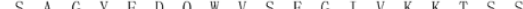
GCCTATTCATCTGCAGGAGGTATATCTGAGTAGgCCATAAGCGCTATTAGAACAGTAAAA \begin{tabular}{llllllllllllllllllll}
$A$ & $Y$ & $S$ & $S$ & $A$ & $G$ & $G$ & $I$ & $S$ & $E$ & $Q$ & $A$ & $I$ & $S$ & $A$ & $I$ & $R$ & $T$ & $V$ & $K$ \\
\hline
\end{tabular} GGTTTAAATGGTTAAGATTTTGATTAAAATAAATATTAAAGCATGATCAAGAaAGCATTC \begin{tabular}{llllllllllllllllllll}
$G$ & $L$ & $N$ & $G$ & $Q$ & $D$ & $F$ & $E$ & $Q$ & $N$ & $K$ & $Y$ & $Q$ & $S$ & $M$ & $I$ & $K$ & $K$ & $A$ & $F$ \\
\hline \hline
\end{tabular}

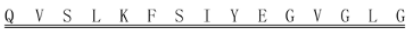

$\underline{\text { ttatcttactcaataaataataaaaaatatcacacagaaaa t }}$ gtatcaaataggttat tagtatttatttgatgtaatagtcaataat tgaactatttttatatttaccaaaagattt t taaactaat taat $t$ t $t$ gaataat tctct $t$ tact taaaagGACTTTAAAATATGATGTT

TTTCTTTGATTTTGCTTTGACTTTTTGGGTAGGATCTAAATTTATTGAAGACGAAGTTA \begin{tabular}{llllllllllllllllllll}
\hline & $F$ & $D$ & $F$ & $A$ & $L$ & $T$ & $F$ & $W$ & $V$ & $G$ & $S$ & $K$ & $F$ & $I$ & $E$ & $D$ & $E$ & $V$ & $Y$ \\
\hline
\end{tabular} TAATCATAATTAAGGAAGAAGCTATAATTTTTCTGATGTATTAACTGCATTTTTAGCAAT \begin{tabular}{llllllllllllllllllll}
$N$ & $H$ & $N$ & $Q$ & $G$ & $R$ & $S$ & $Y$ & $N$ & $F$ & $S$ & $D$ & $V$ & $L$ & $T$ & $A$ & $F$ & $L$ & $A$ & $I$ \\
\hline
\end{tabular} II U S S F E L G Q A U N S I I K A F T Q A TCGTCAAGCTGGATTTACATGTTTTAAgtaaataaattagccagttaattgattttta R Q

aat tagatgtt taaat tat tgct tacaatcaaattaatttttaatat tgettaaaatta ccat tcaatat ttaaaat $t \mathrm{tttcaa}$ aat taaataaaaagATATTAMATAGAAAATCTA

$$
\begin{array}{lllllllllllllllll} 
& \text { I } & L & N & R & K & S & K
\end{array}
$$
aAGTAGATTTAAATGAGAaCGGTATTGACTTAACTAaAaAaTAaATCAATGGaGaAatTA

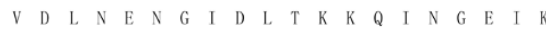
AatTTGAAAATGTAGATTTCTCTTACCCTACTCATCTTGATACAAAGATTCTGAAAAATT $\begin{array}{llllllllllllllllllll}F & E & N & V & D & F & S & Y & P & T & H & L & D & T & K & I & L & K & N & L\end{array}$ TAAATATCTCAATATAACCTCACAAAAAAACAGCTTTTGTAGGTGAATCAGGAAGgtatt $\begin{array}{llllllllllllllllll}N & \text { I S I } & Q & P & H & K & K & T & A & F & V & G & E & S & G & S\end{array}$

ttact taatcctct tctaaaataggatgtat t taaat tt tattttttcaaaat tagTGG

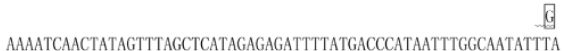

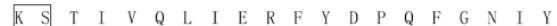
TTTGGATGGTGTCAATCTMAMGGACTTTAMATTAACTTCTCTTCGTTAATCTATCGGATA L D G V N N L K D F K L T S L R R Q S I G Y tgtaggataAgaAcccGitctTtTtgctactactattagagaaAatctattatatggtaA

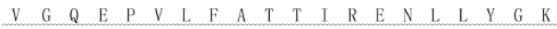
AaGAGATGCAACTGAAGAACAAATGATTGAAGCTCTCAAATAGGCAAACGCTTGGTAATT

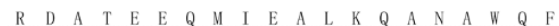
TATTGAAAAATTAGAGAAAGGTTTAGATACTTATGTTGGTACATCTGGTGCTTAATTCTC I E K L L E K G G L D T T Y V G T T S G A Q E F AGGAGGCTAAAAGTAGCGTATTAGCATTGCAAGAGCTATTTTAAAAAATCCAAAAATTCT \begin{tabular}{lllllllllllllllllllll}
\hline$G$ & $G$ & $Q$ & $K$ & $Q$ & $R$ & $I$ & $S$ & $I$ & $A$ & $R$ & $A$ & $I$ & $L$ & $K$ & $N$ & $P$ & $K$ & $I$ & $L$
\end{tabular} TTTGCTTGATGAAGCTACTAGTGCTTTAGACCGTAAAAATGAAGCTCAAATTTAAAGCAC L L D A A T S A L D R K N E A Q I Q S T TTTAGACTCTGTTTCTAMGgatTAaCaACCATCGTtGTAGCCCACAGACtATCAaCaAT

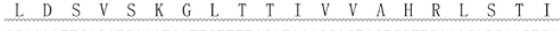
ACAAAATTCAGATGAAATAATTGTTTTAGATAAaGGaGTAGTCGTTGAAGGAGGAACTCA Q N S D E I I I V L D D K G G V V V V E R R G T H TGATGATTTATTAAAAAACAATGGTGCTTATTTTAAATTTGTAGAAAAATAAAAAATAAT D D L L K N N N G A Y Y F K F F V E E K Q K K I I AGAAAAAGAGCAAGAAAAATAAGCTGCTCATAACAATAAAACTTTATGTTTGCTGGGAGA

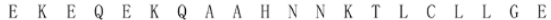
aACATAaTCAagaTtagCATCTTAGTCTTCTTAaACTCTAaATACAGAaCAagaATTTTT
$\begin{array}{llllllllllllllllllll}T & Q & S & R & L & A & S & Q & S & S & Q & T & L & N & T & E & Q & E & F & L\end{array}$ AATGAAGCAGGAAagTTAAAATTAGAATTGCTAAAAATTGATTTAGCAATTTTAAAACT

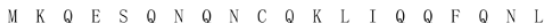
GATAATATTAAAGAagaAgATGATGATAATGATGATGAaGaAGCCTAAAATAAAGCAAT $\begin{array}{llllllllllllllllllll}N & N & I & K & E & E & D & D & D & N & D & D & E & E & A & Q & N & K & A & I\end{array}$ AaGAaGTTCTTTTAGCAGCTAAACTTAAAACACCCCAAAACAAATAGAGATAGTAGAAT $\begin{array}{lllllllllllllllllllll}R & S & S & F & S & S & Q & T & Q & N & T & P & K & Q & I & E & I & V & E & Q\end{array}$ AAATATTATTGATTTATAGAATATTTAGAATACAAATGTGAAGTAAGCCTAAGAAAAgAC $\begin{array}{lllllllllllllllllllll}N & I & I & D & L & Q & N & I & Q & N & T & N & V & K & Q & A & Q & E & K & T\end{array}$ aAACGATTAagGTATTATGAAAGGACTTTTTTCTTATAATGAGAAATAAACCATCAATT

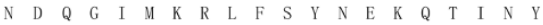
TGTGCTTGGTTTTCTTTTTGCTATTGGCAATGGAGTTTGTTATCCTTTCAGTGGGTACGT \begin{tabular}{lllllllllllllllllllll}
$V$ & $L$ & $G$ & $F$ & $L$ & $F$ & $A$ & $I$ & $G$ & $N$ & $G$ & $V$ & $C$ & $Y$ & $P$ & $F$ & $S$ & $G$ & $Y$ & $V$ \\
\hline
\end{tabular} GCTTGGTAAAATCAGTGATGTGTTGCTTGATAGAACAAGAAGTGATTTTAGAGAACAAAG \begin{tabular}{cccccccccccccccccccc}
$L$ & $G$ & $K$ & $I$ & $S$ & $D$ & $V$ & $L$ & $L$ & $D$ & $R$ & $T$ & $R$ & $S$ & $D$ & $F$ & $R$ & $E$ & $Q$ & $S$ \\
\hline CATTTGGAAAGTCTTTATTTTTTGATTTTGGGTTAGCATAACTCTTGACATGTACTTT
\end{tabular} $\begin{array}{lllllllllllllllllllll}N & L & Q & S & L & Y & F & L & I & L & G & L & A & Q & L & L & T & C & T & F\end{array}$ CTAATTTTACTTCTTCTCGAGAGTAGCTGAATAACTTACATTCAAGTTACGTAAAGATCT

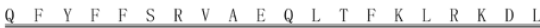
CTTTTAAAAATATTTGAAAATGCCTATTTCTTGGTTTGATCACCCTCATAATACTCCTGG \begin{tabular}{llllllllllllllllllll}
$F$ & $Q$ & $K$ & $Y$ & $L$ & $K$ & $M$ & $P$ & $I$ & $S$ & $W$ & $F$ & $D$ & $H$ & $P$ & $H$ & $N$ & $T$ & $P$ & $G$ \\
\hline
\end{tabular}

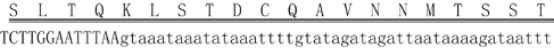
L. G $\quad$ I $\quad$ Q

at tt tat tcaataaagCTTTCTAATGTAAGCTCTTTGGTCAGTGCTTTAGCTTTGGCT \begin{tabular}{lllllllllllllll}
$L$ & $S$ & $N$ & $V$ & $S$ & $S$ & $L$ & $V$ & $S$ & $A$ & $L$ & $A$ & $L$ & $A$ & \\
\hline
\end{tabular} TTTCAGCAGATTGGAGAACAACCCTAGTTGGTTTAAGTCTTATGCCTTTGATGGTTTTGA S A A D W GTTAGGCTTGGTATATGAGCAGAATGGAAGGCTTTGGAGAAAAAACTGATGCCGCATTTA \begin{tabular}{lllllllllllllllllllll}
$Q$ & $A$ & $W$ & $Y$ & $M$ & $S$ & $R$ & $M$ & $E$ & $G$ & $F$ & $G$ & $E$ & $K$ & $T$ & $D$ & $A$ & $A$ & $F$ & $R$ \\
\hline
\end{tabular} \begin{tabular}{llllllllllllllllllll}
$D$ & $S$ & $T$ & $N$ & $M$ & $I$ & $N$ & $E$ & $A$ & $A$ & $C$ & $N$ & $I$ & $R$ & $T$ & $V$ & $T$ & $S$ & $F$ \\
\hline
\end{tabular} GAACAATTAGCAATTAGTCCAAAATTTTACATAAATTTTAGATAAAAATATCAAAGAGA \begin{tabular}{lllllllllllllllllll}
$N$ & $N$ & $Q$ & $Q$ & $L$ & $V$ & $Q$ & $N$ & $F$ & $T$ & $Q$ & $I$ & $L$ & $D$ & $K$ & $N$ & $I$ & $K$ & $E$ \\
\hline TAAAAAAATCTGCTCTGGAaGCAGGTCTTGCTATAGCATGCACAATTTCATTTGTTTG
\end{tabular}

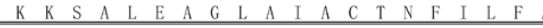
gtaatttcttaatacaaatattaaaaataattaaaattaattattctttatctagCTA TCTATGGTACAATCTTTTATGCAGGATCTACTTTTCATAGGGATTATGATCTTTCGATTG $\begin{array}{llllllllllllllllllll}Y & G & T & I & F & Y & A & G & S & T & F & H & R & D & Y & D & L & S & I\end{array}$ TTGATATGTTTATTTCTATTTAATGCCTAATGTTTGCTGCTATTGGTATAGgtaatatat $\begin{array}{llllllllllllllllll}\text { D } & M & F & \text { I } & S & I & Q & C & \text { L } & \text { M } & F & A & A & \text { I } & G & \text { I } & G\end{array}$

aagatcattttttagagtatctattaagaattttagatacat tt taat ttt tatgtat gaaat tatc t t t tacaaatat tac taat tatcaaaagGCTCAAACAGTCATTATTTGGG $\begin{array}{llllllll}S & N & S & H & Y & L & \text { G }\end{array}$

GATGTAGGTACTTCATAAAATGCTGCAAAGGGTATTTTCTAAGTTCTTGATAGTGTAGAC

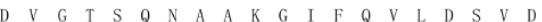
GAAAATCAATTGAATATTTTAAATTTCGATAACCAAGATATTCACGGAGAAATATAATTT

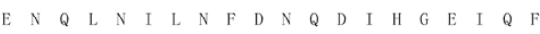
AAGAACGTTACTTTTAAATACCCACAAAGAGATTAGATTATCTTAAAAGATGTTTCTTTT K $N \begin{array}{llllllllllllllllllll} & N & V & T & F & K & Y & P & Q & R & D & Q & I & I & L & K & D & V & S & F\end{array}$ ACCATTCCTGCAGGATAGAAAGTTGCTTTCGTTGGACCTTCTGGAGCAGGCAAATCTTCT \begin{tabular}{llllllllllllllllllll}
\hline & $I$ & $P$ & $A$ & $G$ & $Q$ & $K$ & $V$ & $A$ & $F$ & $V$ & $G$ & $P$ & $S$ & $G$ & $A$ & $G$ & $K$ & $S$ & $S$
\end{tabular}

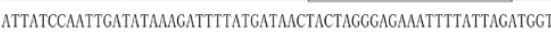
$\begin{array}{lllllllllllllllllllll}I & I & Q & L & I & Q & R & F & Y & D & N & Y & Q & G & E & I & L & L & D & G\end{array}$ GTTGATATAAAGAACTATGATTTGTTGAAATACCGTTCTAAATTTGGAGTTGTATCGTAA

$V \quad D \quad I \quad K \quad N \quad Y \quad D \quad L \quad L \quad K \quad Y \quad R \quad S \quad K \quad F \quad G \quad V \quad V \quad S \quad Q$ GagCCAACACTTTTTACGgGaACTATCAAAGAAAATATAATTTACAATACTGAAAATGTA E P T T L F T T G T I I K E N N I I I Y N MATGAATAATAAATAGAATCCATAACTAMATAAGTAMATGCCTATGATTTCATTACTAAT

$\begin{array}{llllllllllllllllllll}N & E & Q & Q & I & E & S & I & T & K & Q & V & N & A & Y & D & F & I & T & N\end{array}$ TATTCAAAAAgtaagcttttttttctattaatatcctttattaattaatattttttttt Y S K N

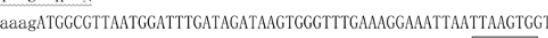
$G \quad V \quad N G F \quad D \quad R \quad Q \quad V \quad G \quad L \quad K \quad G N \quad Q \quad L S G$ GGatagaAataAaGaATTGCTATTTGTAGagctatgatTAaATAaCCAaAaATTATGCTA

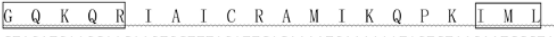
CTAGATGAAGCAACAAGTGCTTTAGATTCACAAAATGAAAAAATAGTCTAAGAATCCCT . D E A T S A L D S Q N E K I V V Q E S S L AACGAAGCAATGAAACAAAAAACTAGTATTTGTGTGGCCCACAGAATATCTACTATTAAA $N$ E A I I K K Q GatTCAGATATGATCTATGTGATGGaAaATGGCAACATAGTCGAGCAagGaAaATATGAT D S D M II I Y Y V N M E E N

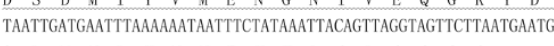
Q L L M N N L K K N N F Y K K L Q Q L G G S S Q aATGAAagAaTGCTATAAAAAAATTATTGACATAACAMAATTAATATTTTAAATTTCTTG TAaAATATATTTTATACATATCAATCTATTATGATTTTAATTTCAGTTATCTTTTAATA TAMATTTAATTAATATATTTAAAAATTC
2520

Figure 3 Full-length and deduced amino acid sequences of Tetrahymena gene abcb15. The transmembrane and nucleotide-binding domains are marked with straight and wavy lines, respectively. Lowercase letters represent intron regions. Walker-A, C-loop, and Walker-B motifs are labeled with boxes. The possible transcription termination signal in the $3^{\prime}$ untranslated region is also labeled with a straight line. 


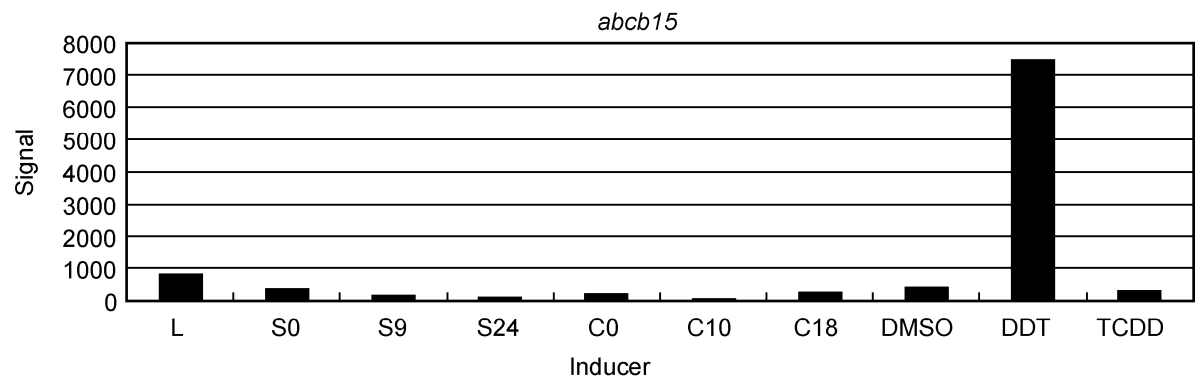

Figure 4 Comparison of the expression levels of $a b c b 15$ during three physiological/developmental stages (growth, starvation, and conjugation) and during treatment with DDT and TCDD. L indicates growing cells with $\sim 1 \times 10^{5}$ cells $\mathrm{mL}^{-1}$. For starvation, $\sim 2 \times 10^{5}$ cells $\mathrm{mL}^{-1}$ cells were collected at 0,9 , and $24 \mathrm{~h}$ (referred to as S0, S9, and S24, respectively). For conjugation, equal volumes of B2086 and CU428 cells were mixed, and samples were collected at 0, 10, and $18 \mathrm{~h}$ after mixing (referred to as $\mathrm{C} 0, \mathrm{C10}$, and C18, respectively) (data from TGED http://tged.ihb.ac.cn/).
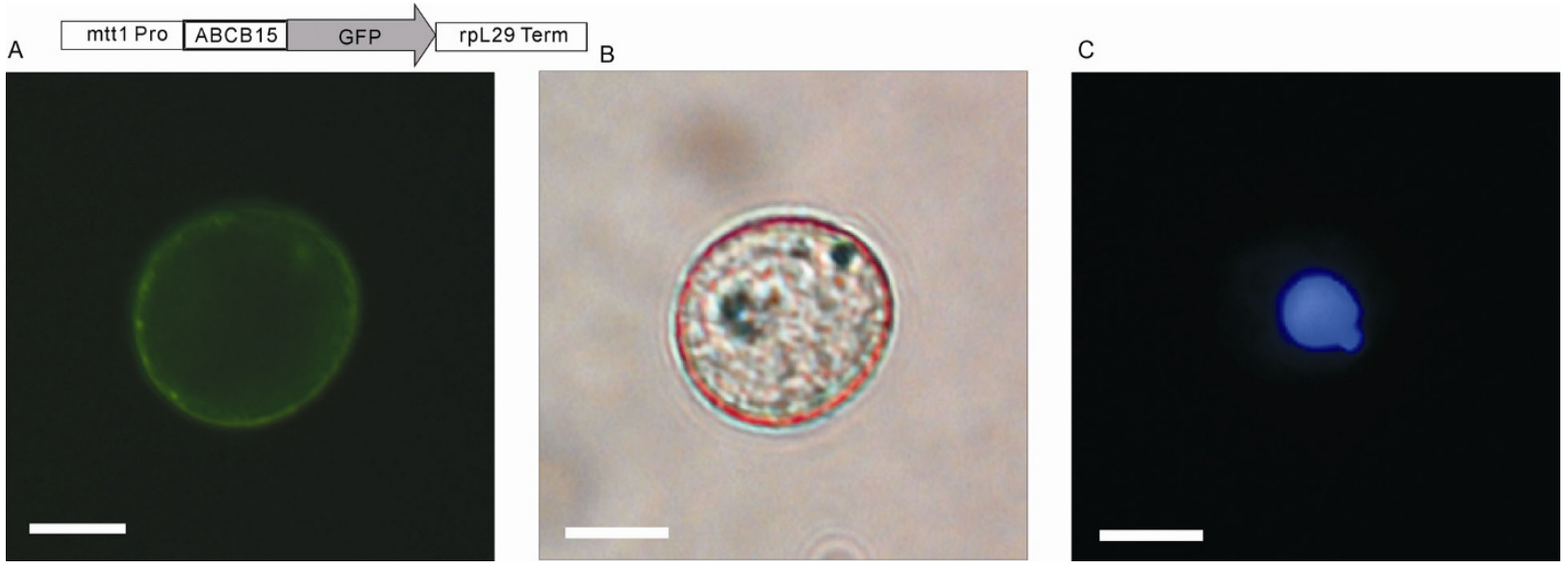

Figure 5 The subcellular localization of ABCB15. A, Fluorescence micrograph of the subcellular localization of ABCB15-GFP expressed from the construct depicted above the micrograph. B, Phase-contrast micrograph of the same cell. C, The macro- and micronucleus of the cell stained with DAPI. Scale bar, $20 \mu \mathrm{m}$.

\subsection{Knock down of abcb15 results in a lower tolerance to DDT in $T$. thermophila}

To analyze the function of $a b c b 15$, we generated the $a b c b 15$ knockdown (abcb15-KD) strain by partially replacing the endogenous $a b c b 15$ gene in the macronucleus of $T$. thermophila with 45 copies of the neo 4 cassette. After treatment with DDT ( $4 \mathrm{mg} \mathrm{L}^{-1}$ ) for $4 \mathrm{~h}$ and $24 \mathrm{~h}$, the expression level of $a b c b 15$ in the $a b c b 15-\mathrm{KD}$ strain was reduced 5-11-fold compared with that in the wild-type (WT) strain using real-time PCR (Figure 6), suggesting that $a b c b 15$ was effectively knocked down by the neo4 cassette in the $a b c b 15-\mathrm{KD}$ strain.

Under conditions of DDT treatment $\left(256 \mathrm{mg} \mathrm{L}^{-1}\right)$, there was no significant difference $(P \geqslant 0.05)$ in growth rate $(k)$ values between the $a b c b 15-\mathrm{KD}$ strain $(k=0.3511)$ and the WT strain $(k=0.3267)$ within $4.5 \mathrm{~h}$ after inoculation, when cells were adjusted to the new environment (Table 1). Since then, cells entered the logarithmic growth period, and the mean generation time (GT) for the $a b c b 15-\mathrm{KD}$ strain $(\mathrm{GT}=3.4951 \mathrm{~h})$ was longer than that for the WT strain $(\mathrm{GT}=2.7232 \mathrm{~h})(P<0.05)$ and reached $14.5 \mathrm{~h}$. This result

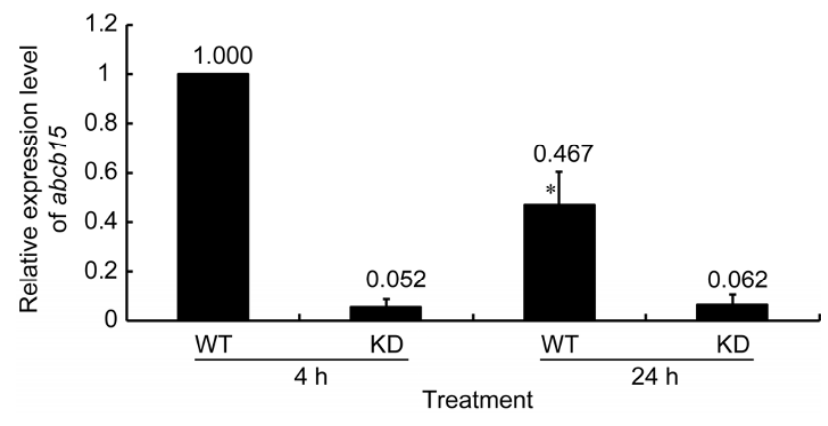

Figure 6 Reduced expression level of $a b c b 15$ analyzed with real-time PCR. The wild-type (WT) and $a b c b 15$ knockdown (KD) strains were treated with $4 \mathrm{mg} \mathrm{L}^{-1}$ for 4 and $24 \mathrm{~h}$, respectively $(n=3)$. ${ }^{*}, P<0.05$.

Table 1 Growth models developed for the lag phase of different strains of $T$. thremophila under $256 \mathrm{mg} \mathrm{mL}^{-1}$ DDT treatment

\begin{tabular}{ccc}
\hline \multirow{2}{*}{ Strains } & \multicolumn{2}{c}{ Growth models } \\
\cline { 2 - 3 } & $0-4.5 \mathrm{~h}$ & $4.5-14.5 \mathrm{~h}$ \\
\hline B2086 & $N_{B}=N_{A} \times \exp (0.3267 \times t)$ & $N_{C}=N_{B} \times 2^{t / 2.7232}$ \\
$a b c b 15-\mathrm{KD}$ & $N_{B}=N_{A} \times \exp (0.3511 \times t)$ & $N_{C}=N_{B} \times 2^{t / 3.4951}$ \\
\hline
\end{tabular}


indicated that the growth rate of the $a b c b 15-\mathrm{KD}$ strain was inhibited by DDT treatment.

\section{Discussion}

In the present study, we showed that in $T$. thermophile, $a b c b 15$ was up regulated by DDT treatment in a specific manner (Figure 4), which is consistent with our previous studies $[8,11]$. The deduced amino acid sequence from our sequencing data revealed that $\mathrm{ABCB} 15$ from $T$. thermophile possesses the typical characteristics of the functional $\mathrm{ABC}$ transporters, i.e., it is formed in a TMD-NBD-TMD-NBD structure. The two TMDs form the translocation pathway and two NBDs hydrolyze ATP. These domains contain a Walker-A motif that binds the nucleotide, a C-loop motif that contacts the nucleotide in the ATP-bound state, and a Walker-B motif that provides a conserved glutamate residue to orchestrate the nucleophilic attack on ATP via a water molecule [24]. Through hydrolyzing ATP to ADP, ABC transporters acquire energy and transport a wide variety of substrates across membranes, such as ions, sugars, amino acids, polypeptides, toxic metabolites and xenobiotics, and drugs. Therefore, these transporters not only provide nutrients for cells, but also protect cells from numerous toxic compounds [25]. Since the ABC transporters are one of the most highly conserved gene super families in prokaryotic and eukaryotic organisms, ranging from bacteria, fungi, and protists to plants and animals, it is very likely the ABCB15 protein from $T$. thermophile also functions in protecting cells from toxic DDT by transporting it out.

The deduced T. thermophile ABCB15 shares a 32.3\% amino acid identity with a human p-glycoprotein (P-gp) encoded by $a b c b 1$, also known as a multidrug resistance $m d r$ gene [26], which is higher than that shared by STE6 in yeast $(26.2 \%)$ [27]. This information suggests that the $T$. thermophile $\mathrm{ABCB} 15$ protein may also function as a $\mathrm{P}$-gp. The human $p$ - $g p$ gene is constitutively present in various normal tissues and expressed primarily in the liver, colon, kidney, adrenal gland, and pancreas. The localization of P-gp mainly on secreting and excretory cells corresponding to its function, i.e., transporting endobiotics and xenobiotics -such as anthracyclines, vinca alkaloids, taxanes, epipodophyllotoxins and topotecan, and protecting cells from toxic agents [28].

It has been shown that human P-gp recognizes DDT as a substrate and performs as an efflux pump to protect cells. For example, $10 \mu \mathrm{mol} \mathrm{L}{ }^{-1}$ DDT treatment resulted in a 2-3-fold increase in the expression level of $m d r l$ in HepG2 cells, and rhodamine staining indicated that DDT was a substrate for the MDR pump. In addition, DDT induced a two-fold increase in $m d r l$ gene expression in HeLa cells, whereas changes in the expression levels of three additional
ABC transporters (bcrp, mrp1, and mrp2) were not detected [29]. In the present study, the expression level of $a b c b 15$ was markedly increased by exposure to $4 \mathrm{mg} \mathrm{L}^{-1}$ DDT, without being increased by a variety of other physiological conditions or by TCDD treatment, indicating that ABCB15 also recognized DDT as a substrate.

As an important membrane pump, the $\mathrm{ABC}$ transporter protein distributes on the plasma membrane and membranes of organelles. Among $11 \mathrm{ABC}$ transporter genes in yeast (Schizosaccharomyces pombe), two were localized on the plasma membrane, two on mitochondrial membrane, four on vacuolar membrane, one on endosomal and Golgi membranes, and two on endoplasmic reticular membrane [30]. Computational analyses indicated that $T$. thermophile $\mathrm{ABCB} 15$ protein was localized on the plasma membrane. In addition, the chimeric ABCB15-GFP protein was evenly distributed in the cell periphery (Figure 5), which is consistent with the results of a study examining ABC transporters in yeast [30]. These results indicate that ABCB15 protein may function as a membrane pump to transport DDT out of the cell and enhance the tolerance of $T$. thermophile to DDT. This hypothesis is supported by our data from $a b c b 15-\mathrm{KD}$ strain. When $a b c b 15$ was knocked down (Figure 6), the tolerance of T. thermophile to DDT exposure decreased, which is manifested by the decreased growth rate.

T. thermophila has two types of nuclei with distinct functions: a micronucleus that contains five pairs of centromere-bearing chromosomes in diploid germlines, and a macronucleus with $\sim 225$ acentromeric chromosomes in somatic nuclei that contain $\sim 45$ copies for most genes [10]. In this study, the $a b c b 15-\mathrm{KD}$ strain was constructed in somatic cells by replacing the neo 4 cassette at the macronucleus $a b c b 15$ coding sequence.

In the presence of $4 \mathrm{mg} \mathrm{L}^{-1} \mathrm{DDT}$, the $a b c b 15-\mathrm{KD}$ strain $(\mathrm{GT}=3.5 \mathrm{~h})$ grew more slowly than WT cells $(\mathrm{GT}=2.7 \mathrm{~h})$, demonstrating that $a b c b 15$ plays an important role in the resistance of $T$. thermophila to DDT. In P-gp ( $a b c b 1)$ knockout mice, cerebral concentrations of amitriptyline and its metabolites were higher than those in controls [31]. Previous studies showed that the following two mechanisms are responsible for the multidrug resistance of human P-gp: (i) TMDs recognize and bind drugs that diffuse into the cell and then transport them out of the membrane, utilizing the energy from the ATP hydrolysis by NBDs, and reduce the concentration of drugs in the cell; (ii) some lipophilic drugs that diffuse very slowly across the lipidic cytoplasmatic membrane are recognized and bound by TMDs and are thus pumped out of the membrane $[32,33]$. The finding that the $a b c b 15-\mathrm{KD}$ strain grew slower than WT cells in the presence of DDT indicates that ABCB15 is a powerful pump that transports DDT out of the cell and protects the cells from the damage caused by DDT. 
1 Eskenazi B, Chevrier J, Rosas LG, Anderson HA, Bornman MS, Bouwman H, Chen A, Cohn BA, de Jager C, Henshel DS, Leipzig F, Leipzig JS, Lorenz EC, Snedeker SM, Stapleton D. The Pine River statement: human health consequences of DDT use. Environ Health Perspect, 2009, 117: 1359-1367

2 Mandavilli A. Health agency backs use of DDT against malaria. Nature, 2006, 443: 250-251

3 Lovett RA. Oceans release DDT from decades ago. Nature, 2010, doi:10.1038/news.2010.4

4 Hemingway J, Hawkes NJ, McCarroll L, Ranson H. The molecular basis of insecticide resistance in mosquitoes. Insect Biochem Mol Biol, 2004, 34: 653-665

5 Hoff RM, Strachan WMJ, Sweet CW, Chan CH, Shackleton M, Bidleman TF, Brice KA, Burniston DA, Cussion S, Gatz DF, Harlin K, Schroeder WH. Atmospheric deposition of toxic chemicals to the Great Lakes: a review of data through 1994. Atmos Environ, 1996, 30: 3505-3527

6 US Department of Health and Human Services. Toxicological Profile for DDT, DDE, and DDD. ATSDR, 2002

7 Eisen JA, Coyne RS, Wu M, Wu D, Thiagarajan M, Wortman JR, Badger JH, Ren Q, Amedeo P, Jones KM, Tallon LJ, Delcher AL, Salzberg SL, Silva JC, Haas BJ, Majoros WH, Farzad M, Carlton JM, Smith RK, Jr Garg J, Pearlman RE, Karrer KM, Sun L, Manning G, Elde NC, Turkewitz AP, Asai DJ, Wilkes DE, Wang Y, Cai H, Collins K, Stewart BA, Lee SR, Wilamowska K, Weinberg Z, Ruzzo WL, Wloga D, Gaertig J, Frankel J, Tsao CC, Gorovsky MA, Keeling PJ, Waller RF, Patron NJ, Cherry JM, Stover NA, Krieger CJ, del Toro C, Ryder HF, Williamson SC, Barbeau RA, Hamilton EP, Orias E. Macronuclear genome sequence of the ciliate Tetrahymena thermophila, a model eukaryote. PLoS Biol, 2006, 4: 1620-1642

8 Miao W, Xiong J, Bowen J, Wang W, Liu YF, Braguinets O, Grigull J, Pearlman RE, Orias E, Gorovsky MA. Microarray analyses of gene expression during the Tetrahymena thermophila life cycle. PLoS One, 2009, 4: e4429

9 Xiong J, Lu XY, Lu YM, Zeng HH, Yuan DX, Feng LF, Chang Y, Bowen J, Gorovsky M, Fu CJ, Miao W. Tetrahymena Gene Expression Database (TGED): a resource of microarray data and co-expression analyses for Tetrahymena. Sci China Life Sci, 2011, 54: 65-67

10 Miao W. Small protozoan Tetrahymena undertakes major tasks. Bull Biol, 2010, 45: 1-4

11 Chang Y, Feng LF, Miao W. Toxicogenomic investigation of Tetrahymena thermophila exposed to dichlorodiphenyltrichloroethane (DDT), tributyltin (TBT), and 2,3,7,8-tetrachlorodibenzo-p-dioxin (TCDD). Sci China Life Sci, 2011, 54: 617-625

12 Miao W, Yu T, Orias E, Wan ML, Fu CJ. Identification of differentially expressed genes in Tetrahymena thermophila in response to dichlorodiphenyltrichloroethane (DDT) by suppression subtractive hybridization. Environ Microbiol, 2006, 8: 1122-1129

13 Feng LF, Miao W, Wu YX. Differentially expressed genes of Tetrahymena thermophila in response to tributyltin (TBT) identified by suppression subtractive hybridization and real time quantitative PCR. Aquat Toxicol, 2007, 81: 99-105

14 Guo LN, Yu T, Feng LF, Miao W. Cloning and expression analysis of Tetrahymena 2-oxoglutarate/malate carrier gene (omc) exposed to dichlorodiphenyltrichloroethane (DDT). Chin J Appl Environ Biol, 2010, 16: 216-221
15 Xiong J, Feng LF, Yuan DX, Fu CJ, Miao W. Genome-wide identification and evolution of ATP-binding cassette transporters in the ciliate Tetrahymena thermophila: a case of functional divergence in a multigene family. BMC Evol Biol, 2010, 10: 330

16 Horton P, Park KJ, Obayashi T, Fujita N, Harada H, Adams-Collier CJ, Nakai K. WoLF PSORT: protein localization predictor. Nucleic Acids Res, 2007, 35(Web Server issue): W585-587

17 Emanuelsson O, Nielsen H, Brunak S, von Heijne G. Predicting subcellular localization of proteins based on their N-terminal amino acid sequence. J Mol Biol, 2000, 300: 1005-1016

18 Yao MC, Yao CH, Halasz LM, Fuller P, Rexer CH, Wang SH, Jain R, Coyne RS, Chalker DL. Identification of novel chromatin-associated proteins involved in programmed genome rearrangements in Tetrahymena. J Cell Sci, 2007, 120: 1978-1989

19 Gaertig J, Kapler G. Transient and stable DNA transformation of Tetrahymena thermophila by electroporation. Methods Cell Biol, 2000, 62: 485-500

20 Mochizuki K. High efficiency transformation of Tetrahymena using a codon-optimized neomycin resistance gene. Gene, 2008, 425: 79-83

21 Bruns PJ, Cassidy-Hanley D. Biolistic transformation of macro- and micronuclei. Methods Cell Biol, 2000, 62: 501-512

22 Whiting RC. Microbial modeling in foods. Crit Rev Food Sci Nutr, 1995, 35: 464-494

23 Boldrin F, Santovito G, Negrisolo E, Piccinni E. Cloning and sequencing of four new metallothionein genes from Tetrahymena thermophila and T. pigmentosa: evolutionary relationships in Tetrahymena MT family. Protist, 2003, 154: 431-442

24 Hollenstein K, Dawson RJ, and Locher KP. Structure and mechanism of ABC transporter proteins. Curr Opin Struct Biol, 2007, 17: $412-418$

25 Holland IB, Blight MA. ABC-ATPases, adaptable energy generators fuelling transmembrane movement of a variety of molecules in organisms from bacteria to humans. J Mol Biol, 1999, 293: 381-399

26 Perez-Tomas R. Multidrug resistance: retrospect and prospects in anti-cancer drug treatment. Curr Med Chem, 2006, 13: 1859-1876

27 McGrath JP, Varshavsky A. The yeast STE6 gene encodes a homologue of the mammalian multidrug resistance P-glycoprotein. Nature, 1989, 340: 400-404

28 Borst P, Elferink RO. Mammalian ABC transporters in health and disease. Annu Rev Biochem, 2002, 71: 537-592

29 Shabbir A, DiStasio S, Zhao J, Cardozo CP, Wolff MS, Caplan AJ. Differential effects of the organochlorine pesticide DDT and its metabolite $p, p^{\prime}$-DDE on p-glycoprotein activity and expression. Toxicol Appl Pharmacol, 2005, 203: 91-98

30 Iwaki T, Giga-Hama Y, Takegawa K. A survey of all 11 ABC transporters in fission yeast: two novel $\mathrm{ABC}$ transporters are required for red pigment accumulation in a Schizosaccharomyces pombe adenine biosynthetic mutant. Microbiology, 2006, 152: 2309-2321

31 Uhr M, Grauer MT, Yassouridis A, Ebinger M. Blood-brain barrier penetration and pharmacokinetics of amitriptyline and its metabolites in p-glycoprotein (abcb1ab) knock-out mice and controls. J Psychiatr Res, 2007, 41: 179-188

32 Baguley BC. Multiple drug resistance mechanisms in cancer. Mol Biotechnol, 2010, 46: 308-316

33 Borowski E, Bontemps-Gracz MM, Piwkowska A. Strategies for overcoming ABC-transporters-mediated multidrug resistance (MDR) of tumor cells. Acta Biochim Pol, 2005, 52: 609-627

Open Access This article is distributed under the terms of the Creative Commons Attribution License which permits any use, distribution, and reproduction in any medium, provided the original author(s) and source are credited. 